Ann. Génét. Sél. anim., I97 I, 3 (4), 487-495.

\title{
EFFETS DE LA CONSANGUINITÉ SUR DEUX GARACTÈRES QUANTITATIFS CHEZ LE VER A SOIE
}

\author{
J.-M. LEGAY \\ Laboratoire de Biométrie, \\ Section de Biologie générale et appliquée. \\ Université Claude-Bernard, \\ 69 - Lyon I
}

\section{RÉSUMÁ}

Les effets d'un système de reproduction consanguin (frère $\times$ sœur) sont étudiés au cours de 7 générations successives chez le Ver à soie, Bombyx mori. Les caractères contrôlés sont le poids de la chrysalide et le poids de soie du cocon chez le mâle et la femelle. Les résultats concernent l'évolution de la variabilité intra- et interfamilles pour chacun des caractères et celle de la corrélation et de la covariance entre ces caractères. La diminution de la variabilité intra-famille (environ la moitié pour un coefficient de consanguinité de 0,250 à 0,672 ) et la relative stabilité de la variance totale sont discutées. L'idée d'une corrélation à variabilité minimale en relation avec l'homozygotie est avancée; en effet la variance de la covariance baisse de 5 à 6 fois (selon le sexe) avec la consanguinité et le coefficient de corrélation final augmente de $50 \mathrm{p}$. 100 dans les deux sexes.

Le nombre de données expérimentales concernant les effets de la consanguinité chez les Insectes, qui constituent pourtant un matériel favorable, n'est pas très grand. En outre sur plusieurs dizaines d'articles publiés, la plupart traitent de caractères de viabilité (fertilité, fécondité, vitesse de développement, pourcentage d'éclosion) ou de caractères morphologiques élémentaires (poids total, taille, longueur d'aile, nombre de soies ou de dents ; quelques études seulement abordent des caractères physiologiques (respiration, adaptation thermique), biochimiques (production d'enzymes) ou comportementaux (vigueur sexuelle, activité migratoire). Enfin la presque totalité des résultats portent sur les Drosophiles (D. melanogaster subobscura et willistoni).

Habituellement les auteurs constatent une dépression des caractères considérés sauf en ce qui concerne certains caractères morphologiques (comme le poids qui reste stable, au moins dans les premières générations) ; ils disent trouver une augmenta- 
tion de la variance totale et une diversification des lignées consanguines. Un résumé de ces résultats et leur interprétation sont donnés par LERNER (I958), dont la revue reste valable encore aujourd'hui.

Il paraît nécessaire d'améliorer nos connaissances en multipliant le nombre d'espèces et le nombre de caractères considérés avant de pouvoir espérer des conclusions nouvelles. C'est pourquoi nous croyons utile de rapporter quelques résultats acquis chez le Ver à soie, Bombyx mori $\mathrm{L}$, sur plusieurs générations d'élevages consanguins, malgré le caractère incomplet de l'information recueillie.

\section{I. - MATÉRIEL, ET MÉTHODE}

Les expériences sur la consanguinité chez Bombyx mori L. rapportées ici ont duré sept ans. L'année $\mathrm{r}$, des pontes de la souche monovoltine $200 \times 300$ ont été prélevées sur une population normale, c'est-à-dire un hybride commercial communément utilisé en France. L'année 2, les individus issus de ces pontes ont été élevés par familles séparées; la reproduction a été effectuée entre frères et sœurs à l'intérieur de chaque famille, si bien que l'année 3 a vu l'élevage de la première génération à coefficient de consanguinité non nul. Les mêmes opérations ont été reproduites les années $4,5,6$ et 7 . Le nombre de familles élevées chaque année a varié selon les moyens matériels dont nous disposions ; tous les individus des familles ont été élevés, mais les contrôles quantitatifs ont été effectués sur des échantillons d'une dizaine de mâles et d'une dizaine de femelles pour chaque famille de la $F_{1}$, sur des échantillons de 20 à 30 individus dans chaque sexe pour chaque famille des générations suivantes.

Les différentes familles se répartissaient en lignées selon l'arbre généalogique représenté à la figure $\mathbf{I}$.

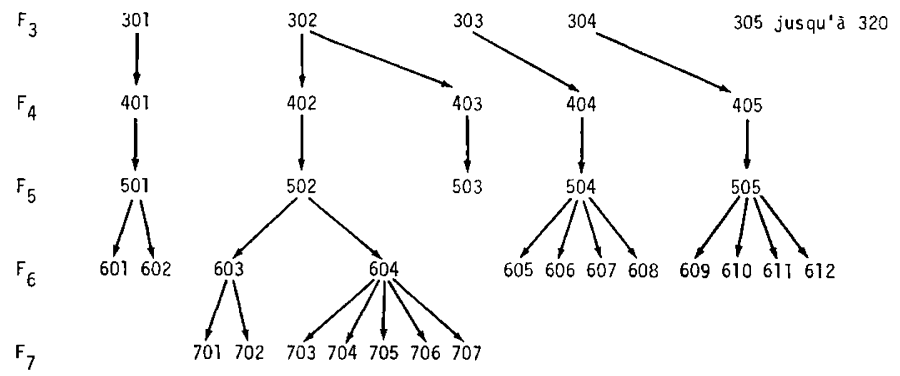

FIG. I. - Arbre généalogique des familles expérimentées

Il est à noter que nous n'avons pas effectué de sélection volontaire dans le choix des géniteurs; ceux-ci, pris dans la zone moyenne du lot qu'ils représentaient, n'ont cependant pas été tirés au hasard. L'apparition de stérilité et de mortalité embryonnaire précoce, surtout dans certaines pontes, a conduit à la disparition de celles-ci. Sans pouvoir présenter sur un petit nombre de générations une véritable étude de ce phénomène, indiquons quelques résultats (tabl. I).

Ces observations ne doivent pas être interprétées globalement, car en fait ce sont certaines lignées qui à chaque génération sont responsables de la plus grande partie de la stérilité ou mortalité constatées.

Le coefficient de consanguinité était calculé selon la formule (FALCONER, I960) :

$$
\mathrm{C}_{\mathrm{F}}=\frac{\mathrm{I}}{4}\left(\mathrm{I}+2 \mathrm{C}_{\mathrm{F}-1}+\mathrm{C}_{\mathrm{F}-2}\right)
$$

où $\mathrm{C}$ est la valeur du coefficient et $\mathrm{F}$ le numéro de la génération.

Le plan expérimental pouvait donc être résumé de la manière donnée dans le tableau 2. 
TABLEAU $I$

Évolution de la stérilité avec la consanguinité

\begin{tabular}{c|c|c|c|c}
\hline \hline \multirow{2}{*}{ Générations } & \multicolumn{3}{|c}{ Pourcentages moyens } \\
\cline { 2 - 5 } & $\begin{array}{c}\text { d'œufs stériles } \\
\text { par ponte }\end{array}$ & $\begin{array}{c}\text { de mortalité } \\
\text { embryonnaire } \\
\text { précoce }\end{array}$ & $\begin{array}{c}\text { de pontes } \\
\text { entièrement stériles }\end{array}$ & $\begin{array}{c}\text { de pontes presque } \\
\text { entièrement stériles }\end{array}$ \\
\hline $\mathrm{F}_{3}$ & 2,0 & 2,0 & 0 & 6,8 \\
$\mathrm{~F}_{4}$ & 8,4 & 23,0 & 0 & 6,0 \\
$\mathrm{~F}_{5}$ & - & - & 12,8 & 4,6 \\
$\mathrm{~F}_{6}$ & 8,4 & 5,2 & 0 & 4,1 \\
$\mathrm{~F}_{7}$ & 7,0 & 7,2 & \\
\hline
\end{tabular}

TABLEAU 2

Plan expérimental

\begin{tabular}{c|c|c|c}
\hline \hline Générations & $\begin{array}{c}\text { Nature } \\
\text { de l'élevage }\end{array}$ & $\begin{array}{c}\text { Nombre } \\
\text { de familles }\end{array}$ & $\begin{array}{c}\text { Coefficient } \\
\text { de consanguinité }\end{array}$ \\
\hline $\mathrm{F}_{1}$ & $\begin{array}{c}\text { Population } \\
\mathrm{F}_{2}\end{array}$ & - & 0 \\
$\mathrm{~F}_{3}$ & Eamilles séparées & 20 & 0 \\
$\mathrm{~F}_{4}$ & Élevage consanguin & 20 & 0,250 \\
$\mathrm{~F}_{5}$ & id. & 5 & 0,375 \\
$\mathrm{~F}_{6}$ & id. & 5 & 0,500 \\
$\mathrm{~F}_{7}$ & id. & 7 & 0,594 \\
\hline
\end{tabular}

Les caractères étudiés étaient le poids de la sécrétion de soie, soie dont on sait qu'elle est tissée en une coque par la chenille juste avant la métamorphose, et d'autre part le poids de la chrysalide. Ces poids étaient mesurés au dixième jour après le début du filage du cocon. Les sexes étaient séparés.

Ces expériences sont donc caractérisées par :

- l'étude simultanée de deux caractères,

- une vitesse élevée d'acquisition de la consanguinité,

- l'absence de sélection volontaire,

- un matériel animal que l'on peut considérer comme domestique.

Les deux caractères considérés (poids de la coque soyeuse, poids de la chrysalide) ont déjà été étudiés quant à leur corrélation (LEGAY, I96I $a$ et I96I $b$ ) et à leur héritabilité (LEGAY, 1962).

\section{II. - RÉSULTATS EXPÉRIMENTAUX}

Les données brutes peuvent être présentées, après un premier traitement, de deux manières selon que l'on veut mettre en évidence la variation des caractères à l'intérieur des familles ou entre familles. Les modalités de calcul ne sont pas évidentes et le procédé que nous allons décrire résulte d'essais préliminaires. 
Le premier calcul consiste à établir les moyennes $x_{i}$ et les écarts-types $\mathrm{S} \bar{x}_{i}$ de ces moyennes pour chaque famille; puis à former pour chaque année la moyenne pondérée $\mathrm{X}_{1}$ et l'écart-type moyen $\mathrm{S}_{1}$, selon les formules :

$$
\mathrm{X}_{1}=\frac{\mathrm{I}}{\mathrm{N}} \sum n_{i} \bar{x}_{i} \quad \mathrm{~S}_{1}^{2}=\frac{\mathrm{I}}{\mathrm{N}} \sum n_{i} \mathrm{~S}_{\bar{x}_{i}}^{2} \quad \text { avec } \mathrm{N}=\sum n_{i}
$$

Le deuxième calcul consiste à former pour chaque année également une moyenne $\mathrm{X}_{2}$ qui ne sera plus pondérée et un écart-type $S_{2}$ de cette moyenne selon les formules:

$$
\mathrm{X}_{2}=\frac{\mathrm{I}}{\mathrm{N}^{\prime}} \sum \bar{x}_{i} \quad \mathrm{~S}^{2}=\frac{\sum\left(\bar{x}_{i}-\mathrm{X}_{2}\right)^{2}}{\mathrm{~N}^{\prime}\left(\mathrm{N}^{\prime}-\mathrm{I}\right)}
$$

en appelant :

$n_{i}$ le nombre d'individus étudiés dans la iieme famille,

$\mathrm{N}$ le nombre total d'individus d'une génération,

$\mathrm{N}^{\prime}$ le nombre de familles d'une génération.

On peut donc présenter les résultats dans le tableau 3 .

TABLEAU 3

Évolution des moyennes et variances en fonction de la consanguinité pour les deux caractères étudiés

\begin{tabular}{|c|c|c|c|c|c|c|c|c|c|c|c|c|}
\hline Génération & \multicolumn{3}{|c|}{$\begin{array}{l}\text { Poids } \\
\text { de la coque mâle }\end{array}$} & \multicolumn{3}{|c|}{$\begin{array}{l}\text { Poids } \\
\text { de la coque femelle }\end{array}$} & \multicolumn{3}{|c|}{$\begin{array}{c}\text { Poids } \\
\text { de la chrysalide mâle }\end{array}$} & \multicolumn{3}{|c|}{$\begin{array}{c}\text { Poids } \\
\text { de la chrysalide femelle }\end{array}$} \\
\hline $\mathrm{F}_{3}$ & $\begin{array}{c}\mathrm{N} \\
191\end{array}$ & $\begin{array}{c}\mathrm{X}_{1} \\
390,70\end{array}$ & $\begin{array}{c}S_{1} \\
15,80\end{array}$ & $\begin{array}{c}\mathrm{N} \\
170\end{array}$ & $\begin{array}{c}\mathrm{X}_{1} \\
404,66\end{array}$ & $\begin{array}{c}S_{1} \\
17,09\end{array}$ & $\begin{array}{c}\mathrm{N} \\
189\end{array}$ & $\begin{array}{c}x_{1} \\
1601,39\end{array}$ & $\begin{array}{c}S_{1} \\
61,64\end{array}$ & $\begin{array}{c}\mathrm{N} \\
169\end{array}$ & $\begin{array}{c}\mathrm{x}_{1} \\
1952,23\end{array}$ & $\begin{array}{c}\mathrm{S}_{1} \\
91,37\end{array}$ \\
\hline$F_{4}$ & 125 & 321,76 & 10,56 & 125 & 342,19 & 10,01 & 125 & 1573,24 & 36,04 & 125 & 2076,24 & 41,73 \\
\hline $\mathrm{F}_{5}$ & 122 & 350,30 & 9,61 & 128 & 376,73 & 9,71 & 122 & 1698,42 & 39,43 & 126 & 2227,18 & 49,71 \\
\hline$F_{6}$ & 230 & 347,19 & 9,07 & 344 & 359,92 & 8,00 & 230 & 1493,61 & 31,51 & 344 & 2016,25 & 33,44 \\
\hline$F_{7}$ & 140 & 340,42 & 7,53 & 210 & 348,96 & 7,28 & 140 & 1468,19 & 30,63 & 210 & 1922,32 & 30,18 \\
\hline
\end{tabular}

A. - Variations à l'intérieur des familles

\begin{tabular}{|c|c|c|c|c|c|c|c|c|c|c|c|c|}
\hline \multirow[t]{2}{*}{ Génération } & \multicolumn{3}{|c|}{$\begin{array}{l}\text { Poids } \\
\text { de la coque mâle }\end{array}$} & \multicolumn{3}{|c|}{$\begin{array}{l}\text { Poids } \\
\text { de la coque femelle }\end{array}$} & \multicolumn{3}{|c|}{$\begin{array}{l}\text { Poids } \\
\text { de la chrysalide mâle }\end{array}$} & \multicolumn{3}{|c|}{$\begin{array}{l}\text { Poids } \\
\text { de la chrysalide femelle }\end{array}$} \\
\hline & $\begin{array}{l}\mathrm{N}^{\prime} \\
20\end{array}$ & $\begin{array}{c}\mathrm{X}_{\mathbf{2}} \\
387,94\end{array}$ & $\begin{array}{l}\mathrm{S}_{2} \\
7,02\end{array}$ & $\begin{array}{l}N^{\prime} \\
20\end{array}$ & $\begin{array}{c}X_{2} \\
406,57\end{array}$ & $\begin{array}{l}\mathrm{S}_{2} \\
8,48\end{array}$ & $\begin{array}{l}N^{\prime} \\
20\end{array}$ & $\frac{X_{2}}{1591,74}$ & $\begin{array}{c}\mathrm{S}_{2} \\
24,81\end{array}$ & $\begin{array}{l}\mathrm{N}^{\prime} \\
20\end{array}$ & $\underset{1957,06}{X_{2}}$ & $\begin{array}{c}\mathrm{S}_{2} \\
45,87\end{array}$ \\
\hline $\mathrm{F}_{4}$ & 5 & 320,65 & 15,35 & 5 & 343,05 & 16,22 & 5 & 1571,45 & 30,77 & $\tilde{5}$ & 2075,72 & 46,09 \\
\hline$F_{5}$ & 5 & 350,64 & 7,46 & 5 & 376,48 & 8,76 & 5 & 1701,54 & 46,21 & 5 & 2227,60 & 52,73 \\
\hline$F_{6}$ & 12 & 344,56 & 13,40 & 12 & 357,09 & 12,28 & 12 & 1491,49 & 42,72 & 12 & 2000,97 & 59,89 \\
\hline$F_{7}$ & 7 & 340,42 & 11,78 & 7 & 348,96 & 14,01 & 7 & 1468,19 & 36,31 & 7 & 1922,31 & 45,50 \\
\hline
\end{tabular}

\section{B. - Variations entre familles}




\section{III. - DISCUSSION. INTERPRÉTATTION}

\section{A. - Étude des deux caractères pris séparément}

On note immédiatement que les caractères eux-mêmes ne varient pas beaucoup et en tout cas pas rapidement en fonction de la consanguinité. En outre, l'ordre de grandeur de ces variations (après plusieurs années de consanguinité) n'est pas sensiblement différent de celui des variations dues au milieu. Rappelons en effet que le Ver à soie dépend, sur le plan nutritionnel, du Mûrier, et que la composition des feuilles de cet arbre est liée à la climatologie locale. Ce résultat est en accord avec ceux notés chez la Drosophile concernant le poids (KIDWELL et KIDWELL, I966) ou la taille du thorax (LINTS, I965).

\section{r. Constatation de l'évolution de la variabilité.}

L'examen du tableau 3 (A) conduit par contre, en ce qui concerne la variabilité des caractères, à une conclusion claire : celle-ci diminue de plus de moitié pendant la durée de l'expérience pour les deux caractères étudiés et dans les deux sexes. Cette diminution est plus forte chez les femelles que chez les mâles pour les deux caractères, elle est maximale pour le poids de la chrysalide femelle. On peut résumer les effets de la consanguinité à l'intérieur des familles en formant un tableau des rapports de 1'année 7 à l'année 3 , les résultats sont donc exprimés en pourcentage de l'année 3 :

$\begin{array}{cc}\text { Rapport } & \text { Rapport } \\ \text { des moyennes } & \begin{array}{c}\text { des écarts-types } \\ -\end{array} \\ 87, \mathrm{I} 3 & - \\ 86,23 & 47,65 \\ 9 \mathrm{I}, 68 & 49,59 \\ 98,46 & 33,03 \\ 95,04 & - \\ 87,55 & -\end{array}$

Le tableau 3 (A) nous permet en outre d'étudier la manière dont s'effectue la diminution de variabilité. Afin de rendre les comparaisons plus faciles, nous avons exprimé l'écart-type en $\mathrm{p}$. roo de la moyenne (coefficients de variation) et présentons les résultats sous forme de graphiques (fig. 2).

On peut aisément conclure que les modifications de la variabilité en fonction de la consanguinité ne sont pas linéaires quels que soient les sexes, et les caractères.

L'examen du tableau 3 (B) et de la figure 3 conduit à remarquer que, si la variabilité augmente, elle le fait de façon fort irrégulière et non parallèle pour les différents caractères. Ainsi l'année 4 paraît anormale pour la variabilité du poids de la coque soyeuse alors qu'elle n'indique rien de semblable pour le poids de la chrysalide, mais nous avons noté au début de cet article une forte mortalité embryonnaire à cette génération, ce qui peut expliquer l'anomalie. 


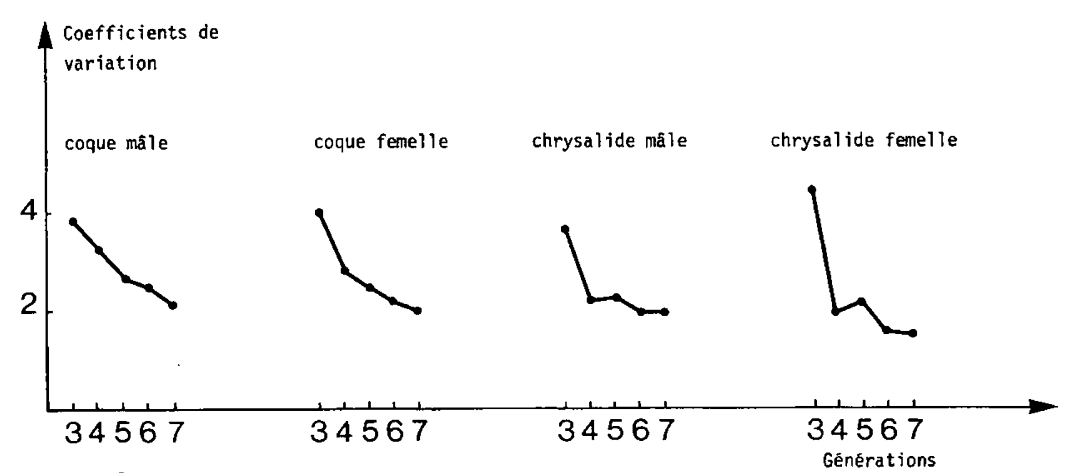

FIG. 2. - Évolution de la variabilité intrafamille en fonction des générations de consanguinité, pour deux caractères quantitatifs, chez Bombyx mori

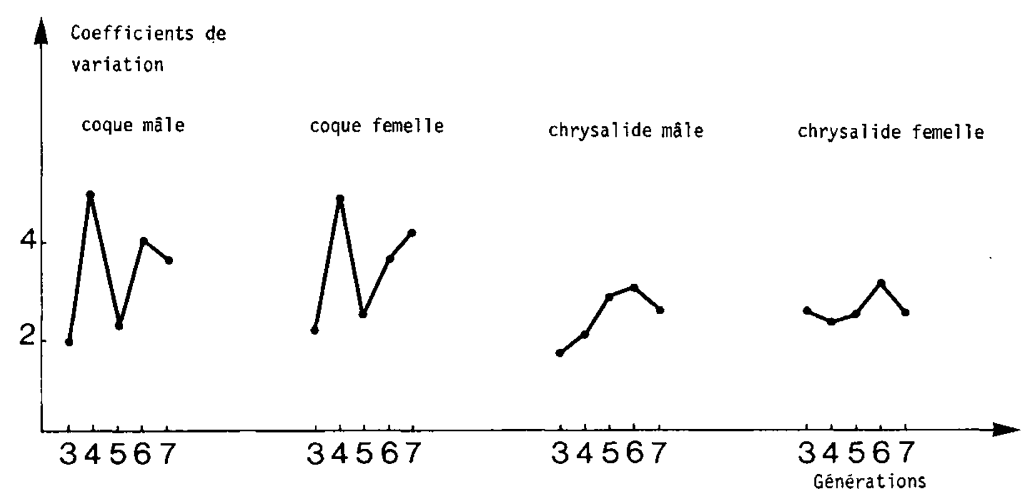

FIG. 3. - Évolution de la variabilité interfamilles en fonction des générations de consanguinité, pour deux caractères quantitatifs, chez Bombyx mori

\section{Diminution de la variabilité intrafamille.}

D'après MALÉCOT (I948), dans le cas des croisements frères-sœurs, le rapport des variances génétiques d'une génération à la précédente doit être $\frac{I+\sqrt{5}}{4}$ c'està-dire de 1'ordre de o,8. La diminution de la variance est donc de type exponentiel. Nous ne disposons que des variances phénotypiques; cependant, si nous admettons que l'héritabilité ne varie pas avec la consanguinité, le rapport des variances génotypiques sera voisin de celui des variances phénotypiques. Si nous calculons ces rapports nous trouvons :

Générations Coque mâle Coque femelle Chrysalide mâle Chrysalide femelle

$\begin{array}{ccccc}- & - & - & - & - \\ 4 / 3 & 0,45 & 0,34 & 0,34 & 0,2 \mathrm{I} \\ 5 / 4 & 0,83 & 0,94 & 1,20 & \mathrm{I}, 42 \\ 6 / 5 & 0,89 & 0,68 & 0,64 & 0,45 \\ 7 / 6 & 0,69 & 0,83 & 0,94 & 0,8 \mathrm{I} \\ \text { Moyennes } & 0,7 \mathrm{I} & 0,70 & 0,78 & 0,72\end{array}$


Malgré une variabilité assez importante, surtout pour le poids de la chrysalide, nous constatons que ces résultats ne sont pas, en moyenne, très éloignés des prévisions théoriques.

\section{Relative stabilité de la variance totale.}

Si l'on additionne pour chaque génération les estimations des variances intra- et interfamilles, on constate une relative stabilité de la variance totale en fonction des générations successives de consanguinité, tout au moins pour le caractère poids de la coque, et ceci quel que soit le sexe. Pour le caractère poids de la chrysalide, la variance totale est maximale à $\mathrm{la}_{\mathrm{a}} \mathrm{F}_{2}$ et, malgré les fluctuations, on peut dire qu'il y a tendance à la diminution. Les deux caractères ne se comportent donc pas de la même manière.

Ainsi pour le poids de la coque dont la variance totale est relativement stable, la consanguinité ne semble pas affecter, jusqu'à la $F_{2}$, les proportions géniques totales, ce qui est cohérent avec les faibles variations des moyennes. Ces résultats vont dans le sens de 1'additivité des gènes concernés par ce caractère.

Pour le poids de la chrysalide, la consanguinité paraît avoir des effets plus rapides et permet de dissocier les facteurs spécifiques de chacun des deux caractères étudiés.

De toute façon, ces résultats sur le Ver à soie ne confirment pas l'augmentation, souvent constatée, de la variance totale avec la consanguinité. Il faudrait peut-être rapprocher cette constatation du caractère domestique du Ver à soie.

\section{B. - Étude des deux caractères pris simultanément}

Ces dernières remarques nous conduisent à l'idée d'examiner les relations entre les deux caractères étudiés et leur évolution en fonction de la consanguinité.

Aussi avons-nous calculé les covariances et les corrélations entre poids de la coque et poids de la chrysalide dans toutes les familles pour chaque génération; il s'agit de corrélations établies à l'intérieur des familles au niveau des individus, donc de même sexe ; nous n'avions pas assez de familles pour calculer des coefficients de corrélations entre familles. Les résultats s'avèrent très intéressants. En effet, les valeurs des covariances ont tendance à diminuer avec la consanguinité, mais pas dans des proportions considérables, mais surtout la variabilité de ces covariances diminue de façon remarquable (dans le rapport de 5 à $\mathrm{I}$ environ). Autrement dit, quels que soient le point de départ et la filiation, la covariance tend vers une valeur relativement stable (tabl. 4).

TABLEAU 4

Évolution des liaisons entre caractères avec la consanguinité

\begin{tabular}{c|c|c|c|c}
\hline \hline \multirow{2}{*}{ Génération } & \multicolumn{2}{|c|}{ Variance de la covariance } & \multicolumn{2}{|c}{ Coefficients de corrélation } \\
\cline { 2 - 4 } & Mâles & Femelles & Mâles & Femelles \\
& & & & \\
\hline & & & & \\
4 & 6335,0 & 5068,1 & 0,34 & 0,40 \\
4 & 4024,7 & 3911,2 & 0,48 & 0,53 \\
5 & 2375,6 & 3642,5 & 0,64 & 0,52 \\
6 & 2400,4 & 2401,0 & 0,67 & 0,66 \\
7 & 1083,9 & 1514,9 & 0,55 & 0,63 \\
\hline
\end{tabular}


Parallèlement les coefficients de corrélation augmentent avec la consanguinité. En passant par le $z$ de Fisher, on peut établir les coefficients de corrélation moyens (tabl. 4).

En outre, seuls les deux coefficients de la génération 7 correspondent à une population homogène de familles (test $\chi^{2}$ appliqué sur les $z$ ). Autrement dit, et en résumé :

la covariance entre les deux caractères diminue avec la consanguinité et tend vers une covariance à variabilité minimale,

la corrélation entre ces mêmes caractères augmente et tend aussi vers une corrélation à variabilité minimale.

Ce résultat est cohérent avec le fait que les variances de chaque caractère diminuent et signifie que les variances diminuent plus vite avec la consanguinité que la covariance.

Notons enfin que l'existence même d'un coefficient de corrélation de l'ordre de 0,6 signifie qu'une partie importante du déterminisme des deux caractères étudiés leur est commun, mais qu'une partie leur est propre et relève peut-être de gènes majeurs, bien que nous ne puissions en donner actuellement la preuve.

Dans une étude sur la stabilité des coefficients de corrélation, et en particulier sur celui défini entre poids de la coque soyeuse et poids de la chrysalide (LEGAY, I96I), nous écrivions :

I ${ }^{\circ}$ que le coefficient de corrélation est une caractéristique de la famille;

$2^{\circ}$ que son déterminisme est en partie génétique.

Nous confirmons et développons ces conclusions. En effet :

$\mathrm{I}^{\circ}$ nous avons vu à quel point la variabilité entre familles pouvait être grande ; à tel point que les valeurs des coefficients de corrélation établies sur les moyennes des familles pour une génération donnée se révèlent très variables,

$2^{\circ}$ les modifications régulières des covariances et corrélations avec la consanguinité montrent bien que la corrélation entre deux caractères est elle-même un caractère héréditaire.

Ainsi donc l'analyse de la variabilité de la corrélation entre deux caractères est possible ; la parenté et la consanguinité sont deux facteurs établis de cette variabilité, plus facile d'accès et d'interprétation dans l'état actuel de nos connaissances que la sélection.

Enfin, du fait que la corrélation entre deux caractères augmente et devient plus stable avec le degré de consanguinité, c'est-à-dire avec une tendance à l'homozygotie, on peut tirer les conclusions suivantes :

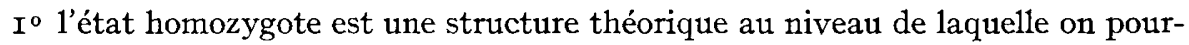
rait définir des coefficients de corrélation de base, à partir desquels pourrait être faite une analyse des écarts avec ces valeurs en fonction de facteurs expérimentaux.

$2^{\circ} 1^{\prime}$ 'état hétérozygote, s'il se confirme qu'il conduit à des corrélations plus faibles entre caractères, comme nous le montrons ici, révèle donc un nouvel avantage ; il permet une adaptation plus souple au milieu puisqu'il autorise deux caractères à varier de façon plus importante l'un par rapport à l'autre.

De toute façon, il serait utile que des études assez larges soient engagées sur les relations possibles entre degré d'hétérozygotie et degré des corrélations entre caractères. 


\section{REMERCIEMENTS}

Je suis heureux de remercier MM. les Professeurs G. MaLÉcot et J. David qui ont accepté de discuter les résultats de cet article et leur interprétation.

\section{SUMMARY}

\section{EFFECTS OF THE INBREEDING ON TWO QUANTITATIVE CHARACTERS IN THE SILKWORM BOMBYX MORI}

The effects of a regular system of inbreeding (full sibbing) are studied during seven generations in the silkworm. The controled characters are the weight of the pupa and the weight of silk of the cocoon on the insects male and female. The results concern the evolution of the variability intra-and interfamilies for each character, and that of the correlation and covariance between these characters. The reduction of the variability intrafamily (about the half for an inbred coeffcient from 0,250 to 0,672 ) and the relative stability of the total variance are discussed. The idea of a correlation with a minimum variability in relation to the homozygosity is advanced; indeed the variance of the covariance decreases between 5 and six time (in relation to the sex) with the degree of inbreeding and the final coefficient of correlation rises of $5^{\circ} \mathrm{p}$. 100 in the two sexes.

\section{RÉFÉRENCES BIBLIOGRAPHIQUES}

FALConer D. S., I960. Introduction to quantitative genetics. The Ronalds Press Company, New York.

Kidwell J. F., Kidwell M. N., I966. The effects of inbreeding on body weight and abdominal chaeta number in Drosophila melanogaster. Canad. J. Genet. Cytol., 8, 207-215.

LEGAY J.-M., I96r a. Sur une corrélation élevée entre coefficients de variation des deux sexes pour un caractère qualitatif chez Bombyx mori. C. R. Acad. Sci. Paris, 252, 342-344.

LEGAY J.-M., I96I $b$. Étude de la stabilité d'une corrélation entre deux caractères quantitatifs. Ann. Eppiphyties, 12, 38I-39r.

LEGAY J.-M., I962. Problèmes posés par l'estimation de l'héritabilité de quelques caractères quantitatifs chez Bombyx mori L. Ann. Génétique, 4, 67-72.

LERNER M., I958. The genetic basis of selection. J. Wiley, New York.

Lints F. A., Lints C. V., I965. Dapression, heterosis and carry-over effects after many generations of inbreeding with Drosophila melanogaster. Genetica, 36, I83-207.

MaLÉcot G., 1948. Les mathématianes de l'hérédité. Masson, Paris. 